\title{
Ring-shaped light illumination ultrasound-modulated optical tomography and its application for sentinel lymph node mapping ex vivo
}

Chulhong Kim, Kwang Hyun Song, Konstantin Maslov, Lihong V. Wang

Chulhong Kim, Kwang Hyun Song, Konstantin Maslov, Lihong V. Wang, "Ring-shaped light illumination ultrasound-modulated optical tomography and its application for sentinel lymph node mapping ex vivo," Proc. SPIE 7177, Photons Plus Ultrasound: Imaging and Sensing 2009, 71771F (24 February 2009); doi: 10.1117/12.808309

SPIE. Event: SPIE BiOS, 2009, San Jose, California, United States 


\title{
Ring-shaped light illumination ultrasound-modulated optical tomography and its application for sentinel lymph node mapping ex vivo
}

\author{
Chulhong Kim, Kwang Hyun Song, Konstantin Maslov, and Lihong V. Wang* \\ Optical Imaging Laboratory, Department of Biomedical Engineering, Washington University in St. \\ Louis, Campus Box 1097, One Brookings Dr. St. Louis, Missouri, 63130
}

\begin{abstract}
We have succeeded in implementing ring-shaped light illumination ultrasound-modulated optical tomography (UOT) in both transmission and reflection modes. These systems used intense acoustic bursts and a charge-coupled device camera-based speckle contrast detection method. By mounting an ultrasound transducer into an optical condenser, we can combine the illuminating light component with the ultrasound transducer. Thus, the UOT system is more clinically applicable than previous orthogonal mode systems. Furthermore, we have successfully imaged an ex vivo methyleneblue-dyed sentinel lymph node (SLN) embedded deeper than $12 \mathrm{~mm}$, the mean depth of human sentinel lymph nodes, in chicken breast tissue. These UOT systems offer several advantages: noninvasiveness, nonionizing radiation, portability, cost-effectiveness, and possibility of combination with ultrasound pulse-echo imaging and photoacoustic imaging. One potential application of the UOT systems is mapping SLNs in axillary staging for breast cancer patients.
\end{abstract}

Keywords: Ultrasound-modulated optical tomography, ring-shaped light illumination, sentinel lymph node biopsy, breast cancer, axillary lymph node dissection.

\section{INTRODUCTION}

Imaging optical properties in the visible and near-infrared regions in biological tissues has become increasingly important because the optical properties are directly related to the functional and morphological parameters of tissues. ${ }^{1,2}$ However, owing to strong light scattering in tissue, keeping high spatial resolution in relatively deep tissue (beyond one transport mean free path) is still a challenge in pure optical imaging techniques such as diffuse optical tomography (DOT). ${ }^{3}$ Ultrasound-aided optical imaging techniques such as photoacoustic (PA) imaging ${ }^{2}$ and ultrasound-modulated optical tomography (UOT) ${ }^{4}$ overcome the drawback of pure optical imaging by taking advantage of optical contrast and ultrasonic spatial resolution. In spite of the great success of PA imaging, the optical contrast of PA imaging is mainly based on optical absorption. However, by supplying information about the optical scattering as well as the optical absorption parameters of tissue, UOT can potentially provide more biological information than PA imaging. ${ }^{5}$

The principles of UOT is that multiply scattered light passing through an ultrasonic focal volume is acoustically phasemodulated by both ultrasound-induced particle displacement and changes in refractive index. By computing the ratio of the ultrasound-modulated light intensity to the unmodulated light intensity at each scanned ultrasonic focal volume, images represent the optical heterogeneities of the tissue. The mechanisms of UOT has been theoretically studied by Leutz et al. ${ }^{6,}$ Wang $^{7}$, and Sakadžic' and Wang ${ }^{8,9}$. Because of the low modulation efficiency and uncorrelated speckle grains, low signal-to-noise ratio (SNR) is a major problem in UOT. A number of detection techniques have been pioneered to detect weak modulated signals efficiently such as parallel speckle detection using a CCD camera ${ }^{10}$, photorefractive crystal based detection ${ }^{11}$, fabry-perot interferometer based detection ${ }^{12}$. Rather than devoting efforts to the detection of weak signals, Kim et al. and Zemp et al. ${ }^{13,14}$ explored the use of intense acoustic bursts as a significant signal enhancement mechanism. Recently, Xu et al. ${ }^{15}$ and Bossy et al. ${ }^{16}$ reported the measurement of mechanical contrast of tissues using UOT in detail. In addition to the low SNR, the practicability of current UOT systems for clinical application is also limited: most current UOT systems are in transmission modes with orthogonal geometries of

\footnotetext{
* Corresponding author: lhwang@biomed.wustl.edu
} 
illumination light, detection light, and ultrasound; tissue samples still need to be immersed in water; and the samples are mechanically moved for scanning ${ }^{10-16}$. Therefore, there is a pressing need to develop a practical UOT system for clinical application. Compared to the orthogonal geometries, ring-shaped light illumination has following benefits. (1) It can reduce the shadow effect of light along the light propagation direction since the light is more evenly distributed. Because of shadows, the previous techniques with CW ultrasound and the orthogonal geometry required image processing to achieve a good quality cross-sectional image. By contrast, the ring geometry can provide a cross-sectional image with less shadow if the imaging plane is parallel to the plane of the donut shape of the illumination. (2) It can be implemented in both transmission and reflection modes. (3) The large area of donut-shaped illumination reduces the optical fluence on the sample surface. (4) The dark-field ring-shaped illumination reduces unwanted surface modulation from outside the ultrasonic focal zone.

For the majority of invasive breast cancers, the surgical removal of primary breast tumor and level I and level II axillary lymph node dissections (ALND) are widely performed. ${ }^{17}$ However, common side effects after ALND include upperextremity lymphedema, arm numbness, impaired shoulder mobility, arm weakness, and infections in the breast, chest, or arm. ${ }^{18}$ Therefore, as a less invasive alternative to ALND, sentinel lymph node biopsy (SLNB), that is, biopsy of the first lymph node receiving drainage from a cancer-containing area of the breast, has become increasingly important in breast cancer treatment. ${ }^{19}$ By reducing the number of ALND, the risk of side effects can be alleviated. ${ }^{20}$ Although SLNB with methylene blue dye and radioactive tracers has an identification rate of $90-95 \%$ and a sensitivity of 88 $95 \%,{ }^{21-23}$ the widely used detection of gamma radiation using a gamma camera is ionizing and intraoperative. ${ }^{24}$ Recently, in vivo non-ionizing photoacoutic imaging has been applied non-invasively to detect SLNs containing methylene blue dye in a rat model. ${ }^{25}$ Since UOT like photoacoustic imaging is able to image optical contrast with high ultrasonic spatial resolution, non-ionizing UOT has the potential to detect methylene-blue-dyed SLNs non-invasively. In addition, thanks to the use of a relatively cost effective and small continuous wave $(\mathrm{CW})$ diode laser, the UOT can potentially be clinically more attractive for the detection of SLNs than photoacoustic imaging, which usually requires a bulky and relatively expensive pulsed laser. In this Proceedings artile, we have implemented a ring-shaped light illumination UOT system in both transmission and reflection modes, using intense acoustic bursts and a charge-coupled device (CCD) camera-based speckle contrast detection technique. ${ }^{13}$ We can implement a portable and relatively economical UOT system by mounting the light source (a cw diode laser), the light detector (a CCD camera), and the ultrasonic transducer on a scanning stage. For more clinical practicability, the UOT system itself moves instead of the sample, and the sample is positioned below a water tank, rather than immersed in the reflection mode system. In a preliminary test for clinical applicability, we have successfully imaged an ex vivo methylene-blue-dyed sentinel lymph node (SLN) buried deeper than $12 \mathrm{~mm}$, the mean depth of the top surface of human SLNs, in chicken breast tissue.

\section{EXPERIMENT}

Figure 1 shows the transmission mode system. The ring-shaped light illumination was formed sequentially by a planoconcave lens, a spherical conical lens, and an optical condenser made of acrylic sheet. This illumination has an advantage over previous illumination geometries: it can reduce the shadow effect of light along the light propagation direction since the light is more evenly distributed. Because of shadows, the previous transmission-mode-technique with $\mathrm{CW}$ ultrasound required image processing to achieve a good quality cross-sectional image. By contrast, the ring geometry can provide a cross-sectional image with less shadow if the imaging plane is parallel to the plane of the donut shape of the illumination. To make the system compact and relatively inexpensive, we utilized a small size diode laser (Melles Griot, 56ICS153/HS; 657-nm wavelength; $9 \times 6 \times 13 \mathrm{~cm}$ along the $X, Y$, and $Z$ axes) mounted on the same post as the optics. A narrowband, high-power, focused ultrasound transducer was placed into the middle of the optical condenser. The transducer was partially immersed in water inside a tank which had an opening at the bottom sealed with a thin, disposable, clear membrane. The ultrasonic focus was aligned with the line focus of light in water. A CCD camera (Basler, A312f; 12-bit, 640 $\times 480$ pixels) was positioned underneath the water tank, and the main axis of the camera was aligned with the ultrasound propagation axis. 


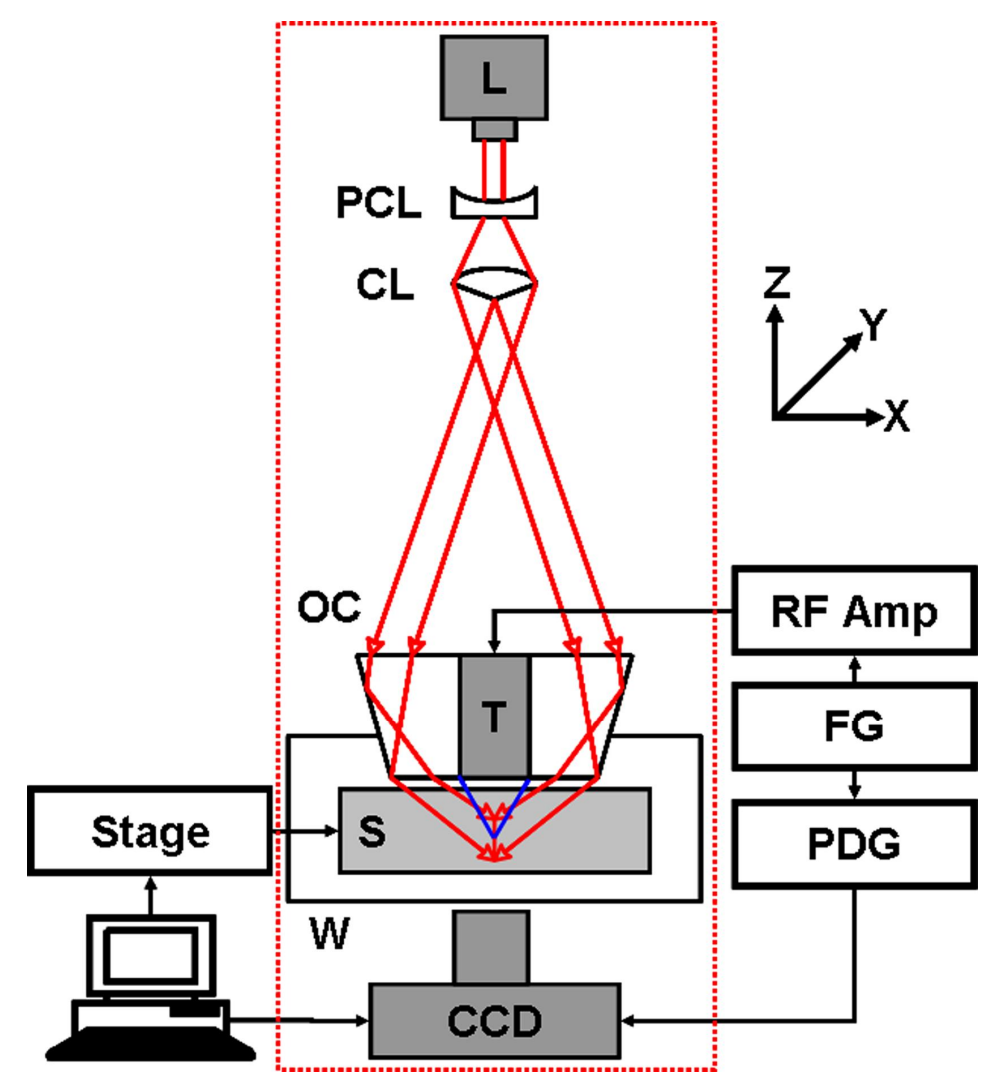

Figure 1: The experimental schematic of transmission-mode ring-shaped light illumination UOT system. L; laser, PCL; planoconcave lens, CL; conical lens, OC; optical condenser, T; ultrasound transducer, S; sample, W; water tank, RF amp; RF amplifier, FG; function generator, PDG; pulse delay generator, CCD; CCD camera.

Figure 2 shows the reflection mode system. The ring-shaped light illumination is formed sequentially by a planoconcave lens, a spherical conical lens, and an optical condenser composed of two plano-convex lenses to enhance the collecting efficiency of diffusively reflected light. The concept of light illumination and application of ultrasound was similar to the transmission mode system. Compared with a coaxial bright-field light illumination design, this configuration has the following advantages: First, the large area of donut-shaped illumination reduces the optical fluence on the sample surface to $8 \mathrm{~mW} / \mathrm{cm}^{2}$, within the current safety limit. Second, thanks to the evenly distributed light illumination, the light shadow effect can be alleviated. Third, the dark-field ring-shaped illumination reduces unwanted surface modulation from outside the ultrasonic focal zone. A CCD camera was also positioned on the scanner, so diffusely reflected light from the tissue sample first traveled back through the optical condenser, was reflected by a mirror, and was collected by the CCD camera. A CCD camera (Basler, A312f; 12-bit, $640 \times 480$ pixels) captured speckle patterns emerging from the sample. By placing a lens tube, which acted as an iris, in front of the CCD camera, we matched the average speckle size to a single CCD pixel size. Ultrasonic bursts were synthesized by a function generator (Agilent, 33250A) and amplified by an RF amplifier (Amplifier Research, 75A250), and subsequently drove the ultrasound transducer. We used a low $0.83 \mathrm{~Hz}$ duty cycle to prevent damage to the transducer. The function generator triggered a pulse-delay generator (Stanford Research, DG535) that produced two CCD trigger pulses for each burst. The burst synchronized speckle pattern was captured with ultrasound; then, another one was captured without ultrasound. The exposure time of the CCD camera was equivalent to the duration of an ultrasonic burst. The laser speckle contrast was computed with and without ultrasound modulation. The UOT signal was defined as the change in speckle contrast between ultrasound on and off (off value - on value). 


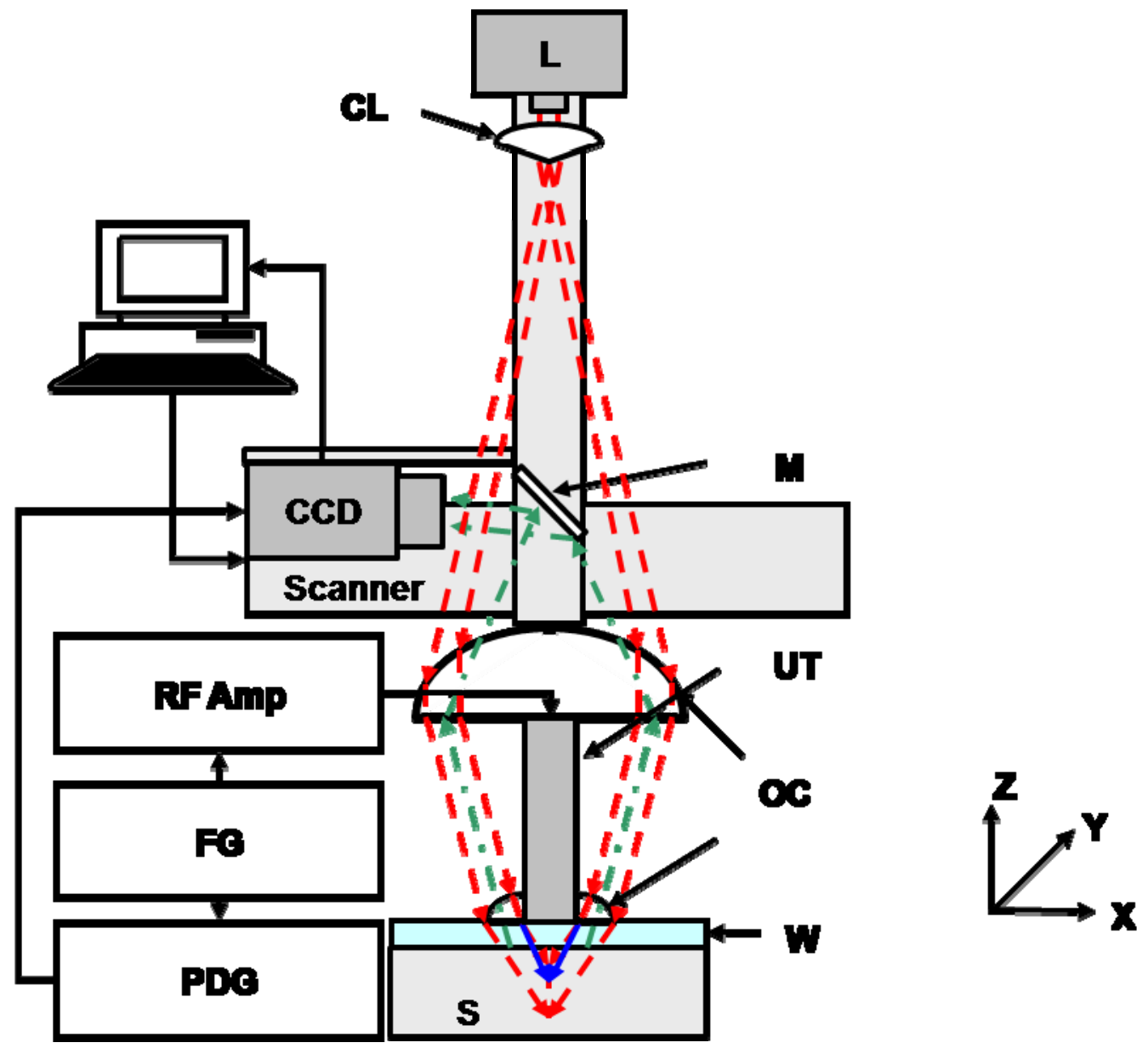

Figure 2: The experimental schematic of reflection-mode ring-shaped light illumination UOT system. L; laser, CL; conical lens, M; mirror, OC; optical condenser, UT; ultrasound transducer, S; sample, W; water tank, RF amp; RF amplifier, FG; function generator, PDG; pulse delay generator, CCD; CCD camera.

\section{RESULTS AND DISCUSSIONS}

To investigate the potential for cross-sectional imaging using these UOT systems, we scanned 1-cm thick tissue phantoms that contained optically absorptive patterned objects. The phantoms were made of $10 \%$ gelatin by weight and $1 \%$ intra-lipid by volume, and their reduced scattering coefficient were $\sim 9 \mathrm{~cm}^{-1}$. The thickness of the patterned shape along the ultrasound propagation direction was about $1 \mathrm{~mm}$. An acoustic peak pressure of $1.6 \mathrm{MPa}$ was applied to the samples. First, using the transmission mode UOT system, we imaged an optically patterned object in Figure 3 . The patterned shape is clearly resolvable in Fig. $3 \mathrm{~b}$ and matches well with the photograph in Fig. 3a. The spatial resolution, defined as the one-way distance across the $25 \%$ and $75 \%$ points between the maximum and minimum of the edge spread function, is $870 \pm 59 \mu \mathrm{m}$ (standard error). The SNR is $199 \pm 13$ (standard error). 

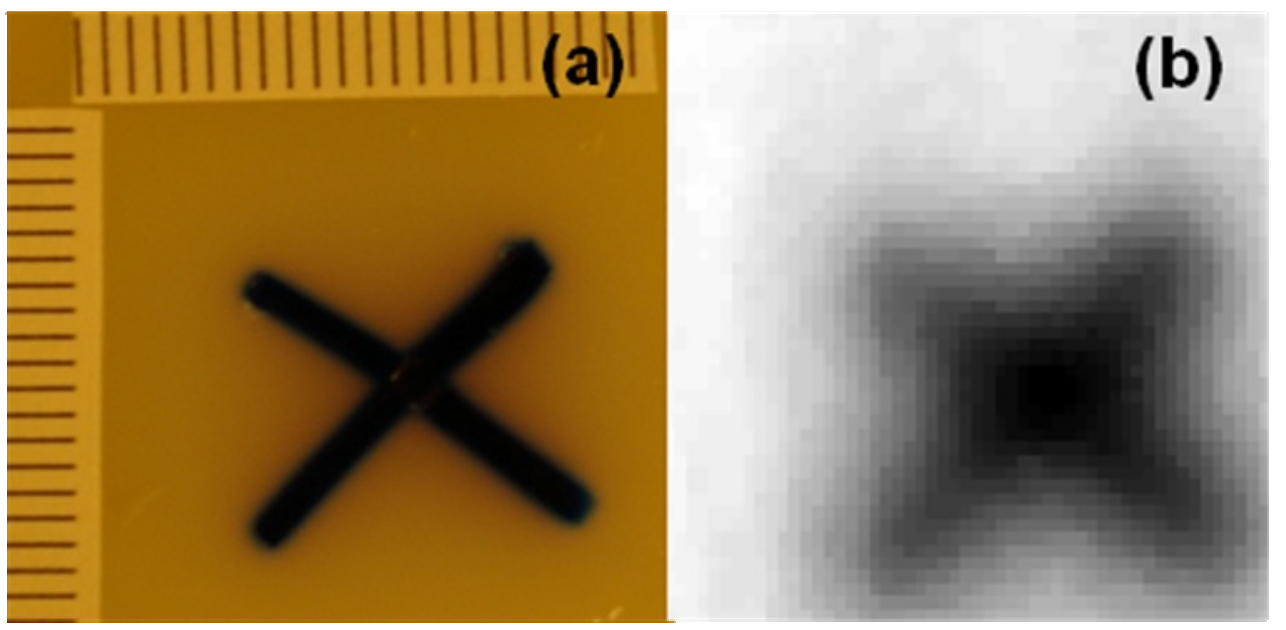

Figure 3: An UOT image of an optically patterned object using the transmission-mode UOT system. (a) Photograph of an optically absorptive target. (b) UOT image.

Next, using the reflection mode UOT system, we imaged an optically absorptive target in Figure 4. The object in the photograph, Fig. 4(a), is clearly imaged using the reflection mode UOT system in Fig. 4(b). The spatial resolution, defined as the one-way distance between the $25 \%$ and $75 \%$ points of the edge spread function, is $1.16 \pm 0.23 \mathrm{~mm}$ (standard error). The SNR, defined as the ratio of the mean to the standard deviation of the UOT signals, is $32 \pm 3$ (standard error). This SNR is about 6 times worse than the reported SNR from a transmission-mode ring-shaped light illumination UOT system $(199 \pm 13$ (standard error)) since the collection of diffusely reflected light passing back through the optical condenser is much less efficient than the collection of diffusely transmitted light passing directly through the sample.
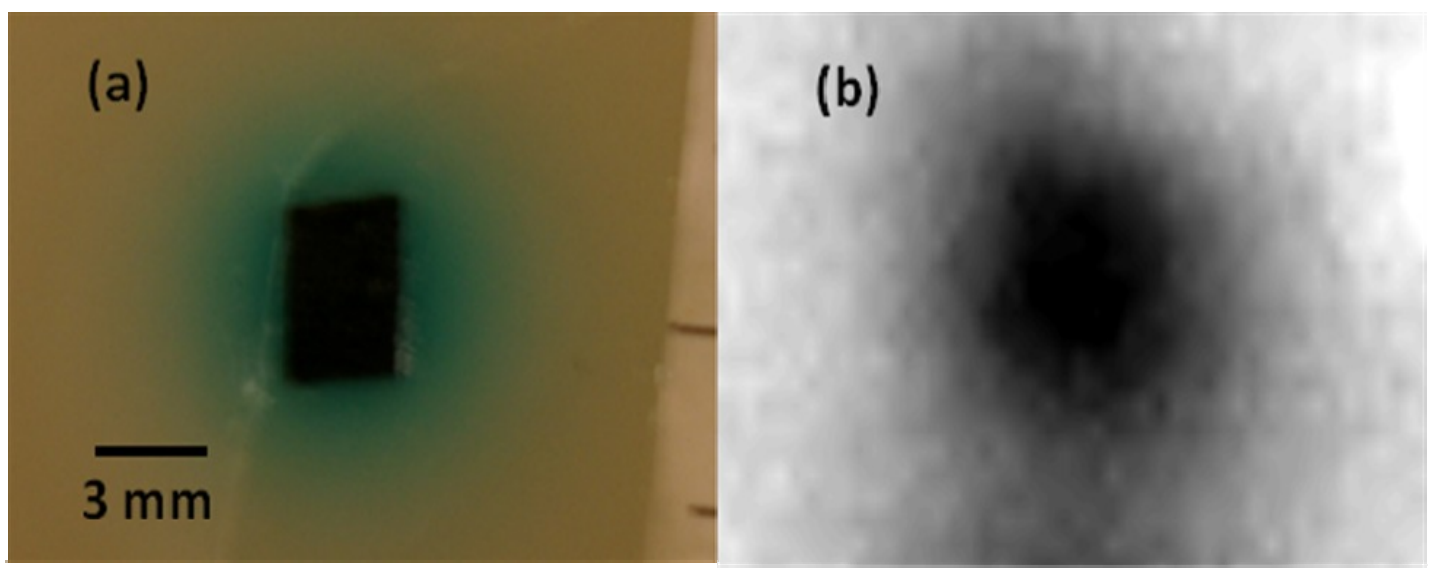

Figure 4: An UOT image of an optically patterned object using the reflection-mode UOT system. (a) Photograph of an optically absorptive target. (b) UOT image.

To show the feasibility of imaging SLNs containing methylene blue dye with the transmission mode system, we scanned $1.2 \mathrm{~cm}$ thick chicken breast tissues containing two excised lymph nodes. First, an intradermal injection of 0.12 $\mathrm{ml}$ of $1 \%$ methylene blue dye $(10 \mathrm{mg} / \mathrm{mL}$, American Regent, INC) was performed on the right forepaw pad of an adult male Sprague Dawley rat weighing $300 \mathrm{~g}$. About 15 minutes after administration of the methylene blue dye, the animal was euthanized with an overdose of pentobarbital. One methylene-blue-dyed lymph node was excised from the right side of the animal and another one from the left side (without any dye) in Fig 5a. The two excised nodes were buried between two chicken slices. Only the methylene-blue-dyed node was clearly seen in Fig 5b. 


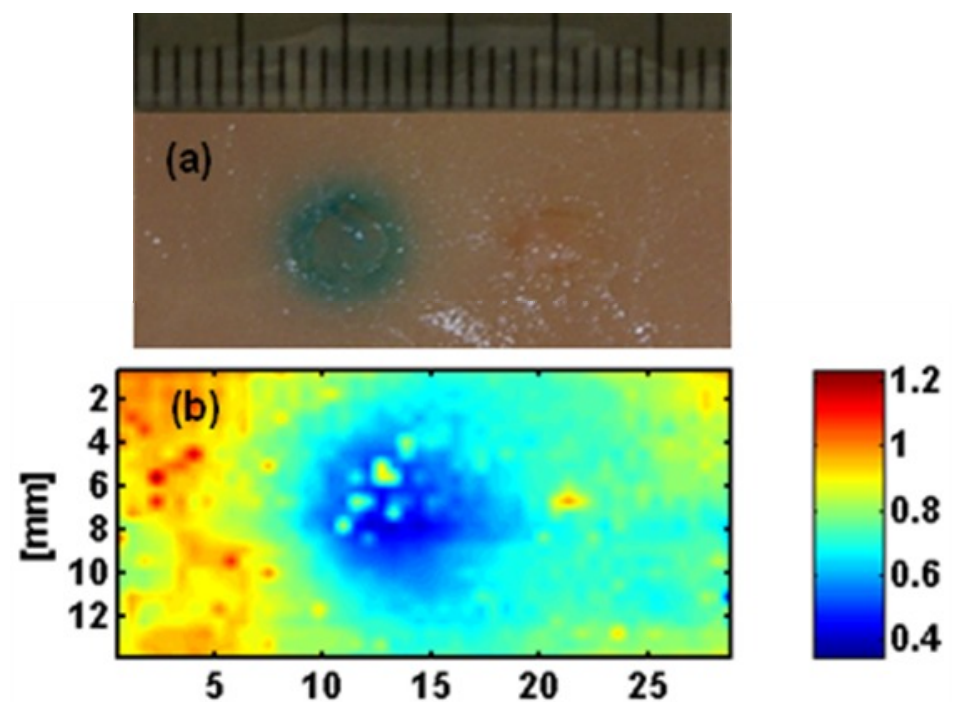

Figuire 5. Ex vivo cross-sectional images of two sentinel lymph nodes in $1.2 \mathrm{~cm}$ thick chicken breast tissue using the transmission-mode UOT system. (a) Photograph of two nodes; the left node is dyed with methylene blue and the right one is not. (b) UOT image.

To examine the feasibility of this technology for clinical application, the total thickness of chicken tissue was increased up to $3.2 \mathrm{~cm}$, and the experiment was repeated. The mean depth of human sentinel lymph nodes is about $1.2 \pm 0.5 \mathrm{~cm}$ in ultrasonography. Figure 6a shows two excised lymph nodes in chicken tissues, and the total thickness is about $3.2 \mathrm{~cm}$ (Fig. 6b). Only the methylene-blue-dyed node was clearly seen in UOT image (Fig. 6c), whereas the light intensity based image does not show any information (Fig. 6d).



Figuire 6. Ex vivo cross-sectional images of two sentinel lymph nodes in $3.2 \mathrm{~cm}$ thick chicken breast tissue using the transmission-mode UOT system. (a) Photograph of two nodes; the left node is dyed with methylene blue and the right one is not. (b) Photograph of the thickness of two chicken slices. (c) UOT image. (d) Light intensity based image.

In addition, using the reflection-mode system, we imaged an ex vivo methylene-blue-dyed SLN embedded 10 mm deep in chicken breast tissue. First, $0.25 \mathrm{ml}$ of $1 \%$ methylene blue dye $(10 \mathrm{mg} / \mathrm{mL}$, American Regent, INC.) was injected intradermally on the right forepaw pad of an adult male Sprague Dawley rat weighing $380 \mathrm{~g}$. The animal was euthanized by administering an overdose of pentobarbital 15 minutes after the methylene blue injection. One 
methylene-blue-dyed node was removed from the right axilla of the animal and another from the left side (without any dye) in Fig. 7a. Only the methylene-blue-dyed node was clearly seen in UOT image (Fig. 7b). To confirm the SLN, we performed a photoacoustic imaging, which has a similar configuration to the UOT system. Only methylene-dyed node was clearly seen in the PA image (Fig. 7c). Furthermore, the unmodulated light intensity based image did not provide any information about the localized methylene-blue-dyed SLN (Fig. 7d).

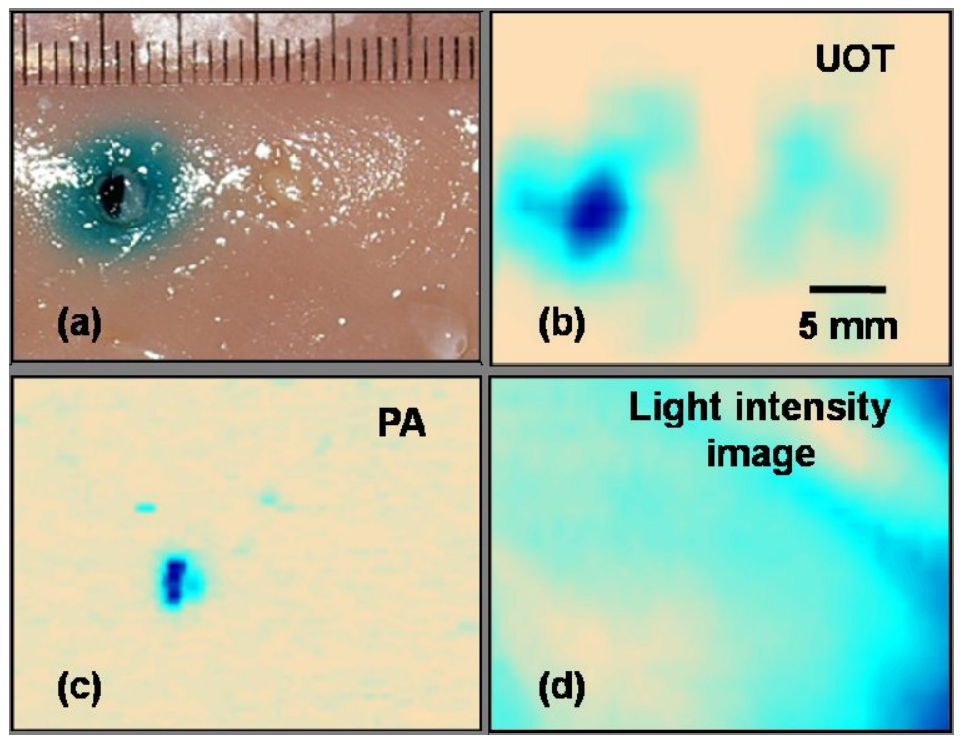

Figuire 6. Ex vivo cross-sectional images of two sentinel lymph nodes buried $1.0 \mathrm{~cm}$ deep in chicken breast tissue using the reflection-mode UOT system. (a) Photograph of two nodes; the left node is dyed with methylene blue and the right one is not. (b) UOT image. (c) PA image. (d) Light intensity based image.

\section{CONCLUSIONS}

In conclusion, we have implemented both transmission and reflection modes ring-shaped light illumination ultrasoundmodulated optical tomography and applied the systems to image an ex vivo methylene-blue-dyed SLN buried in biological tissue. These UOT systems have several advantages: 1. Compared to the previous orthogonal mode transmission systems, these UOT systems are more practical for clinical applications. 2. Compared to the coaxial brightfield light illumination system, they have less light shadow effect due to the even light distribution, and it reduces unwanted surface modulation. 3. Compared to photoacoustic imaging, it is more portable and cheaper thanks to the small and cheap light source. It also provides optical scattering information. 4. It is nonionizing and noninvasive, unlike ionizing and intraoperative gamma radiation based detection technique for mapping SLNs. 5. It offers the possibility of combining three imaging techniques: UOT, PA imaging, and single-element ultrasound pulse-echo imaging. On the basis of above features and the preliminary experimental results, it is expected that this technique can clinically and practically be tested to image methylene-blue dyed SLNs in humans in vivo.

\section{ACKNOWLEDGEMENT}

This research was supported by NIH Grant Nos. R33 CA 094267, R01 NS46214, and R01 CA106728.

\section{REFERENCES}

[1] J. G. Fujimoto, "Optical coherence tomography for ultrahigh resolution in vivo imaging," Nat Biotechnol. 21(11), 1361-1367 (2003). 
[2] H. F. Zhang, K. Maslov, G. Stoica, and L. V. Wang, "Functional photoacoustic microscopy for high-resolution and noninvasive in vivo imaging," Nat Biotechnol. 24(7), 848-851 (2006).

[3] B. W. Zeff, B. R. White, H. Dehghani, B. L. Schlaggar, and J. P. Culver, "Retinotopic mapping of adult human visual cortex with high-density diffuse optical tomography," Proc Natl Acad Sci USA. 104(29), 12169-12174 (2007).

[4] L. Wang, S. L. Jacques, and X. Zhao, "Continuous-wave ultrasonic modulation of scattered laser light to image objects in turbid media," Opt Lett. 20(6), 629-631 (1995).

[5] S. R. Kothapalli, S. Sakadzic, C. Kim, and L. V. Wang, "Imaging optically scattering objects with ultrasoundmodulated optical tomography," Opt Lett. 32(16), 2351-2353 (2007).

[6] W. Leutz and G. Maret, "Ultrasonic modulation of multiply scattered light," Phys. B 204, pp. 14-19 (1995).

[7] L. V. Wang, "Mechanisms of ultrasonic modulation of multiply scattered coherent light: an analytic model," Phys. Rev. Lett. 87, (043093) pp. 1-4 (2001).

[8] S. Sakadzic and L. Wang, "Correlation transfer and diffusion of ultrasound-modulated multiply scattered light," Phys. Rev. Lett. 96, 163902 pp. 1-4 (2006)

[9] S. Sakadzic and L. V. Wang, "Correlation transfer equation for ultrasound-modulated multiply scattered light," Phys. Rev. E. 74, pp. 036618 1-10 (2006).

[10] S. Leveque, A. C. Boccara, M. Lebec, and H. Saint-Jalmes, "Ultrasonic tagging of photon paths in scattering media: parallel speckle modulation processing," Opt. Lett. 24, pp. 181-183 (1999).

[11] S. Sakadžic' and L. V. Wang, "High-resolution ultrasound-modulated optical tomography in biological tissues," Opt. Lett. 29, pp. 2770-2772 (2004).

[12] T. W. Murray, L. Sui, G. Maguluri, R. A. Roy, A. Nieva, F. Blonigen, C. A. DiMarzio, "Detection of ultrasoundmodulated photons in diffuse media using the photorefractive effect," Opt. Lett. 29, pp. 2509-2511 (2004).

[13] C. Kim, R. J. Zemp, and L. V. Wang, "Intense acoustic bursts as a signal-enhancement mechanism in ultrasoundmodulated optical tomography," Opt. Lett. 31, pp. 2423-2425 (2006).

[14] R. J. Zemp, S. Sakadz ic', and L. V. Wang, "Stochastic explanation of speckle contrast detection in ultrasoundmodulated optical tomography,” Phys. Rev. E. 73, pp. 061920 1-5 (2006).

[15] X. Xu, H. Zhang, D. Qing, C. Kim, P. Hemmer, L. V. Wang, "Photorefractive detection of tissue optical and mechanical properties by ultrasound modulated optical tomography,” Opt. Lett. 32, pp. 656-658 (2007).

[16] E. Bossy, A. R. Funke, K. Daoudi, and A. Boccara, "Transient optoelastography in optically diffusive media," Appl. Phys. Lett. 90, pp. 174111.1-174111.3 (2007).

[17] Early stage breast cancer. Consens Statement., 8:1-19 (1990).

[18] K. K. Swenson M. J. Nissen, C. Ceronsky, L. Swenson, M. W. Lee, and T. M. Tuttle, "Comparison of side effects between sentinel lymph node and axillary lymph node dissection for breast cancer," Annals of Surgical Oncology., 9, 745 (2002).

[19] D. Krag, D. Weaver, T. Ashikaga, et al., "The sentinel node in breast cancer - a multicenter validation study," N. Engl. J. Med., 339, 941 (1998).

[20] K. M. McMasters, T. M. Tuttle, D. J. Carlson, et al., "Sentinel lymph node biopsy for breast cancer: a suitable alternative to routine axillary dissection in multi-institutional practice when optimal technique is used," J. Clin. Oncol.,18, 2560 (2000).

[21] O. A. Ung, "Australian experience and trials in sentinel lymph node biopsy: the RACS SNAC trial," Asian J Surg., 27, 284 (2004).

[22] D. Nejc, M. Wrzesien, J. Piekarski, J. Olszewski, P. Pluta, J. Kus'mierek, A. Jeziorskia, "Sentinel node biopsy in patients with breast cancer - evaluation of exposure to radiation of medical staff," The Journal of Cancer Surgery, 32, 133 (2006).

[23] K. H. Song, E. Stein, J. Margenthaler, and L. V. Wang, "Noninvasive photoacoustic sentinel lymph node imaging using methylene blue in a rat model," J. Biomed. Opt., 13, 54033 (2008). 